\title{
Quantized Absorption Strength of Multi-Exciton Quantum Dots
}

\author{
K. Wójcik, A. Grodecka And A. Wóss \\ Institute of Physics, Wrocław University of Technology \\ Wybrzeże Wyspiańskiego 27, 50-370 Wrocław, Poland
}

Energy and absorption/recombination spectra of up to two electronhole pairs confined in a spherical quantum dot are studied numerically as a function of dot radius (i.e., confinement volume). The transition between fermionic and bosonic behavior of the confined excitons is identified in coincidence with enhancement of low-energy absorption strength.

PACS numbers: 73.22.-f, 78.67.Hc, 71.35.-y

\section{Introduction}

Being bound states of two fermions, the excitons $(\mathrm{X}=\mathrm{e}+\mathrm{h})$ can in some situations behave like bosons [1, 2]. A spectacular example is their Bose-Einstein condensation $[3,4]$, recently demonstrated $[5]$ in a two-dimensional coupled quantum well. However, when excitons are confined in small quantum dots [6], spatial quantization of their e and $\mathrm{h}$ constituents exceeds excitonic binding. In consequence, the multi-exciton ground states in such systems are determined by Pauli exclusion principle applied to the individual fermionic constituents [7]. This can be expressed in terms of effective Pauli blocking for the excitons themselves, observed experimentally in photoluminescence studies showing population of consecutive excitonic shells as a function of increasing excitation power [8]. E.g., the lowest $(s-)$ shell of a spherical dot can only hold two excitons (including spin), the next $(p-)$ shell can hold six excitons, etc. In contrast, the allowed population of the exciton ground state without confinement is macroscopic (proportional to the real-space volume). Motivated by recent modulation reflectivity experiments [9], in this paper we study transition between those two extremes, i.e., collapse of the exciton Pauli blocking effect as a function of the dot size. In particular we seek a signature of this collapse in enhancement of the ground-state oscillator strength (i.e., low-energy absorption).

\section{Model}

We use a very simple model for a quantum dot with a variable volume (or area): an ideal spherical surface of radius $R$. While we concentrate on a 
general effect, this model might be adequate to two-component semiconductor nanocrystals (with material with a narrower band gap deposited in a form of a thin layer around a spherical nanocrystal of a material with a larger band gap). The dot radius $R$ is to be compared with a characteristic dimension of an exciton, i.e., effective Bohr radius $a_{\mathrm{B}}=\epsilon \hbar^{2} / \mu e^{2}$. Here, $\mu=\left(m_{\mathrm{e}}^{-1}+m_{\mathrm{h}}^{-1}\right)^{-1}=m_{\mathrm{e}} /\left(1+r^{-1}\right)$ is the exciton reduced mass for a given mass ratio $r=m_{\mathrm{h}} / m_{\mathrm{e}}$. This leads to a dimensionless dot size, $s=R / a_{\mathrm{B}}$. The characteristic quantization energies $\varepsilon_{l}=\hbar^{2} l(l+1) / 2 m_{\alpha} R^{2}$ ( $\alpha=\mathrm{e}$ or $\mathrm{h}$ ) must be compared to the effective excitonic Rydberg, $R y=e^{2} / 2 \epsilon a_{\mathrm{B}}$, yielding, e.g. $\varepsilon_{1}^{(\mathrm{e})} / R y=2 s^{-2} /\left(1+r^{-1}\right)$.

The one- and two-exciton spectra are obtained from exact diagonalization of $\mathrm{e}+\mathrm{h}$ or $2 \mathrm{e}+2 \mathrm{~h}$ Coulomb Hamiltonians in the configuration-interaction (CI) basis. The single-particle states are standard spherical harmonics $Y_{l}^{m}(\theta, \phi)$, labeled by angular momentum quantum numbers $l$ and $m$, allowing for analytical expression of the two-body matrix elements. The $\mathrm{X}$ and $2 \mathrm{X}$ eigenstates are labeled by total angular momentum $L$, related to the wave vector $k=L / R$. In such numerical calculation the major source of error is the restriction of single-particle basis to a number of lowest shells $\left(l \leq l_{\max }\right)$, accurate only for sufficiently small $s$.

\section{Results and discussion}

\subsection{One exciton}

We begin with one exciton. The energy spectra for $r=1$ and different values of $s$ are shown in Fig. 1. For $s=\frac{1}{4}$ the low-energy spectrum can be predicted from addition of e and $\mathrm{h}$ angular momenta, assuming both particles in their low-energy shells (although due to the $r=1$ symmetry, degeneracy of the $\mathrm{e} \leftrightarrow \mathrm{h}$ symmetric states is removed by interaction at arbitrarily small $s$ ). This is the small dot regime. For $s=5$ (vanishing confinement regime) the lowest-energy band is a well-developed exciton dispersion. The intermediate frames show the transition.

(a) $R / a_{B}=1 / 4$

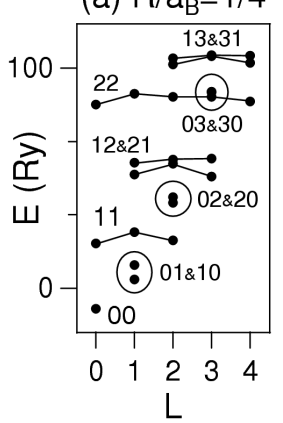

(b) $R / a_{B}=1 / 2$

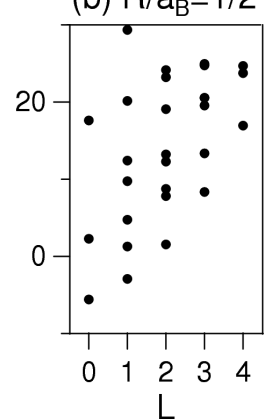

(c) $R / a_{B}=1$

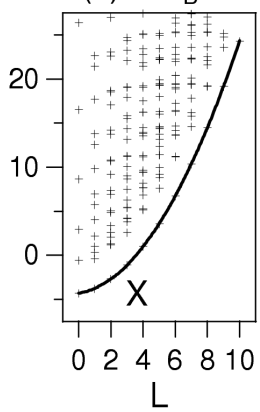

(d) $R / a_{B}=5$

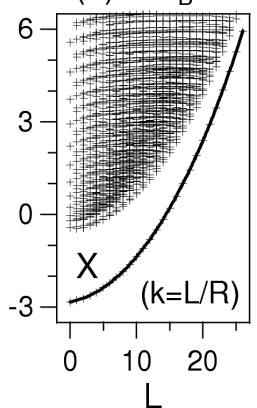

Fig. 1. Energy spectra of one $\mathrm{e}-\mathrm{h}$ pair in spherical quantum dots of different radii $R$, calculated for the mass ratio $m_{\mathrm{h}} / m_{\mathrm{e}}=1$ and including single-particle angular momentum shells with $l \leq 15$. Ry is Rydberg; $a_{\mathrm{B}}$ is Bohr radius. In (a) the lowest eigenstates are connected into bands labeled by e and $\mathrm{h}$ angular momenta $\left(l_{\mathrm{e}} l_{\mathrm{h}}\right)$. 
In Fig. 2 we show similar spectra for $r=5$. Broken e $\leftrightarrow$ h symmetry rearranges the energy spectrum of a small dot, but the familiar excitonic dispersion of Fig. 1a is restored when the confinement is removed.

(a) $\mathrm{R} / \mathrm{a}_{\mathrm{B}}=1 / 4$

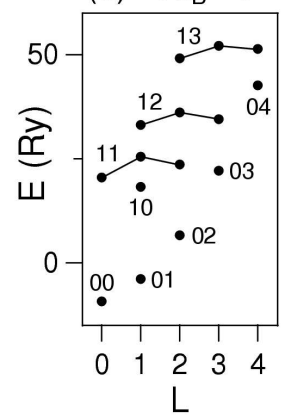

(b) $R / a_{B}=1 / 2$

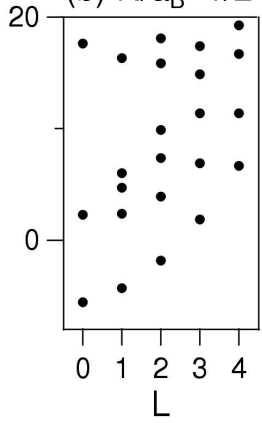

(c) $R / a_{B}=1$

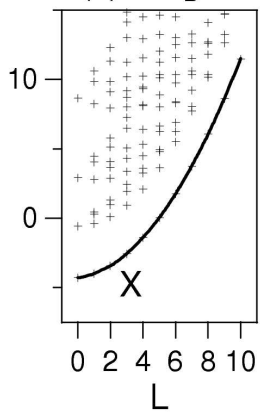

(d) $\mathrm{R} / \mathrm{a}_{\mathrm{B}}=5$

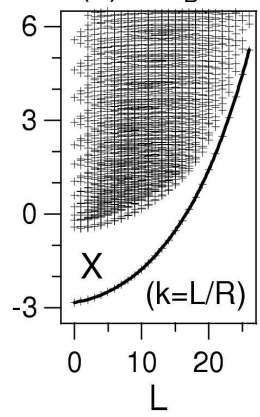

Fig. 2. The same as Fig. 1, but for the mass ratio $m_{\mathrm{h}} / m_{\mathrm{e}}=5$.

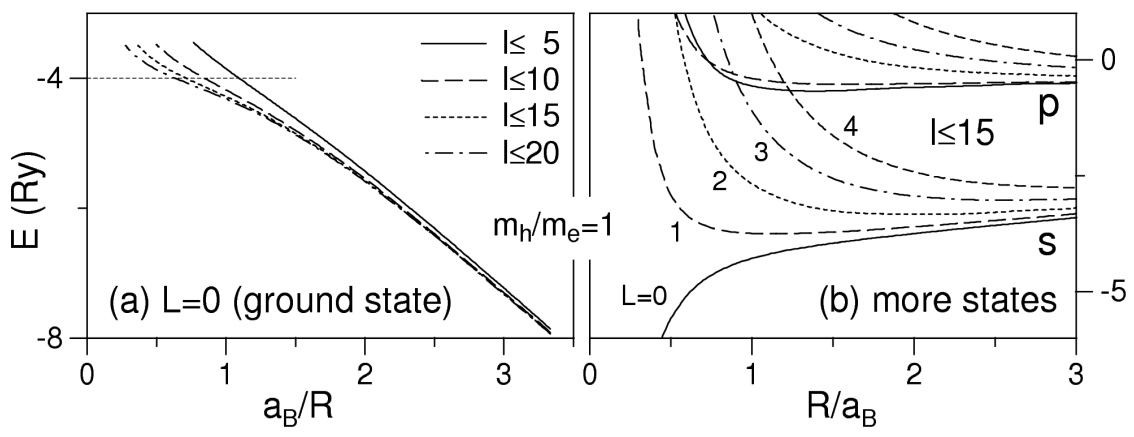

Fig. 3. (a) Ground state energy $E$ of an $\mathrm{e}-\mathrm{h}$ pair as a function of inverse dot radius $R^{-1}$, calculated for $m_{\mathrm{h}}=m_{\mathrm{e}}$ and for different numbers of included angular momentum shells. (b) Size dependence of different energy levels obtained for $l \leq 15$.

The continuous size-dependence of the energy levels is shown in Fig. 3. For $s^{-1} \rightarrow 0$ the ground state energy must converge to $-4 R y$ of a free $2 \mathrm{D}$ exciton. Figure 3 a gives an idea about the number of shells that must be included in a CI calculation at a given $s$. Clearly, while the overall structure of the spectra in Figs. 1d and 2d appear correct, their absolute energy positions are very inaccurate. Figure $3 \mathrm{~b}$ shows the development of excitonic $s$ and $p$ states when confinement weakens (within each band, different $L$ 's refer to the center-of-mass motion).

The excitonic oscillator strengths $\omega$ are shown in Fig. 4. Only the $L=0$ states are optically active. In small dots, the distribution of $\omega$ among different $L=0$ states (i.e., among the shells) is determined by overlaps of the relevant single-particle e and $\mathrm{h}$ orbitals. When confinement weakens, the excitonic correlation 


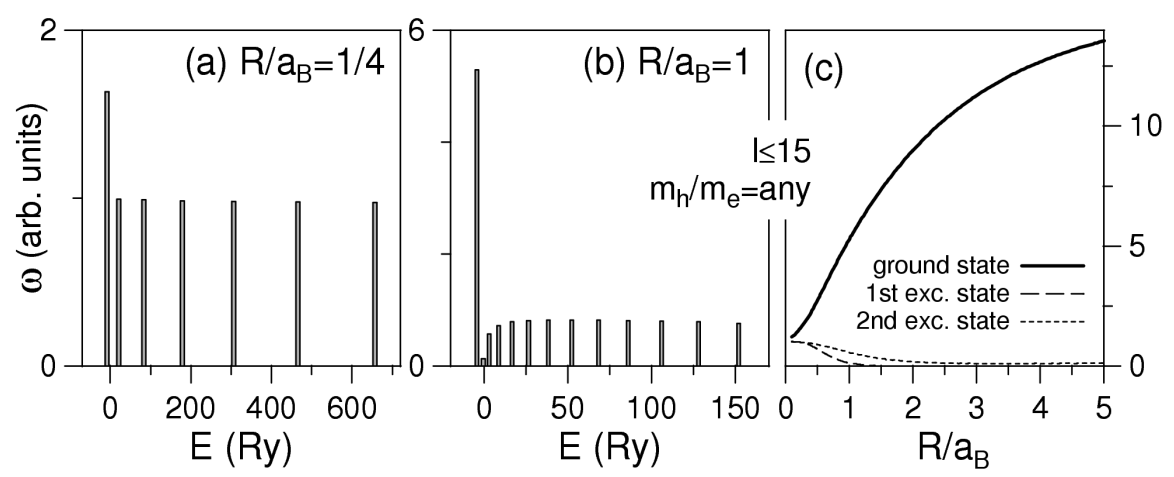

Fig. 4. (a,b) Absorption spectra (oscillator strength $\omega$ vs. energy $E$ ) of one $\mathrm{e}-\mathrm{h}$ pair in spherical dots of different radii $R$. (c) Dependence of oscillator strengths for the three lowest optically active $(L=0)$ states on $\operatorname{dot}$ radius $R$.

emerges in the $L=0$ ground state, to which also most of the oscillator strength is continuously transferred from the higher states. This also leads to the strong enhancement of the ground state.

\subsection{Two excitons}

The $2 \mathrm{e}+2 \mathrm{~h}$ energy spectra for different dot sizes $s$ are shown in Fig. $5(r=1$, symmetric case) and Fig. $6(r=5$, more realistic case). As for a single exciton, only in small dots the spectra are very sensitive to the mass ratio $r$ (and they can be understood by addition of angular momenta of four constituent fermions). However, in contrast to a single-exciton, recombination in a coupled two-exciton system can now occur also from $L \neq 0$ states. The oscillator strengths $\omega$ are indicated in Figs. 5 and 6 by diameters of circles drawn around each energy level.
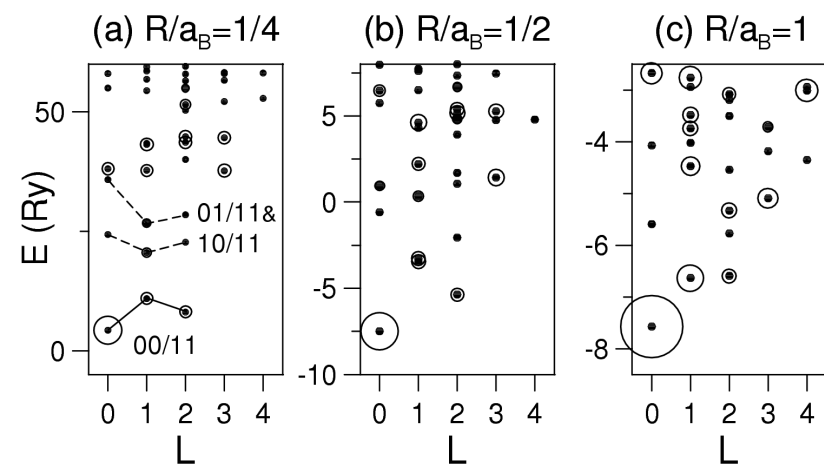

(d) $R / a_{B}=5$

Fig. 5. Energy spectra of two $\mathrm{e}-\mathrm{h}$ pairs in spherical quantum dots of different radii $R$, calculated for $m_{\mathrm{h}}=m_{\mathrm{e}}$ and including angular momentum shells with $l \leq 6$. Oscillator strength $\omega$ of each state is proportional to circle diameter. In (a), lowest eigenstates are labeled by e and h angular momenta $\left(l_{\mathrm{e}} l_{\mathrm{h}} / l_{\mathrm{e}}^{\prime} l_{\mathrm{h}}^{\prime}\right)$. 
(a) $R / a_{B}=1 / 4$

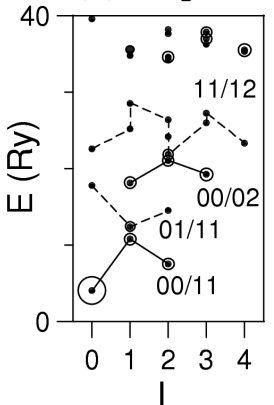

(b) $R / a_{B}=1 / 2$

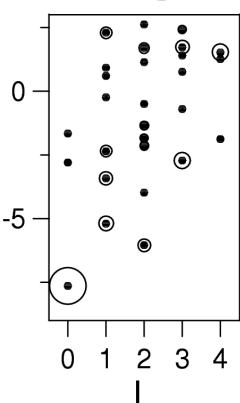

(c) $R / a_{B}=1$

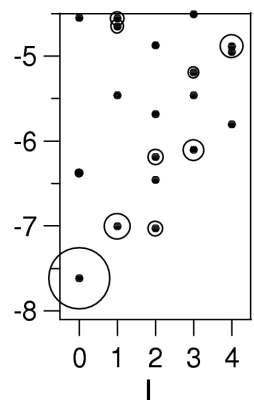

L (d) $\mathrm{R} / \mathrm{a}_{\mathrm{B}}=5$

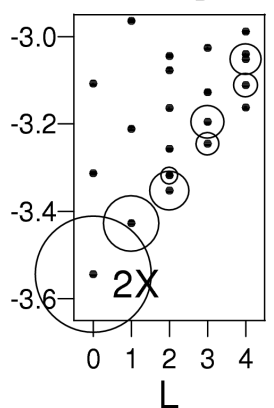

Fig. 6. The same as Fig. 5, but for the mass ratio $m_{\mathrm{h}} / m_{\mathrm{e}}=5$.
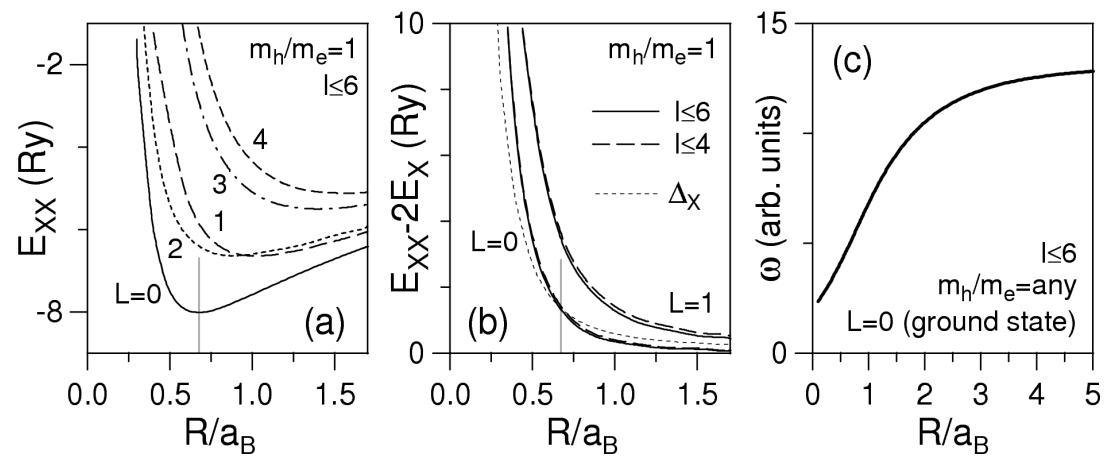

Fig. 7. (a) Lowest energy levels $E$ at different angular momenta $L$ of two e-h pairs as a function of dot radius $R$, calculated for $m_{\mathrm{h}}=m_{\mathrm{e}}$ and $l \leq 6$. (b) Two-pair energies $E$ measured from two one-pair ground state energies $E_{\mathrm{X}}$ for $l \leq 4$ and $l \leq 6$. (c) Size dependence of oscillator strength $\omega$ of the two-pair ground state.

The continuous size-dependences extracted from those spectra is presented in Fig. 7. Frame (a) shows the size-evolution of the lowest energy levels at a few different angular momenta. Competition of kinetic energy and $\mathrm{e}-\mathrm{h}$ attraction leads to a minimum in ground state energy $E_{\mathrm{XX}}$ at $s \approx 0.65$. Frame (b) shows $\delta_{\mathrm{XX}}=E_{\mathrm{XX}}-2 E_{\mathrm{X}}$ to compare one- and two-exciton ground states. This quantity is also compared with the excitation gap of a single exciton, $\Delta_{\mathrm{X}}=E_{\mathrm{X}}^{*}-E_{\mathrm{X}}$ (here, $E_{\mathrm{X}}^{*}$ is the energy of the exciton's first excited state). Coincidentally, the value of $s \approx 0.65$ determines the dot size beyond which $\delta_{\mathrm{XX}}<\Delta_{\mathrm{X}}$, i.e., $E_{\mathrm{XX}}<E_{\mathrm{X}}+E_{\mathrm{X}}^{*}$. This marks onset of effective attraction between excitons filling two lowest single-exciton states. Alternatively, this can be interpreted as crossover into the regime in which excitons no longer obey the effective Pauli exclusion principle and begin to populate single-exciton states like (weakly interacting) bosons. Frame (c) shows an increase of the ground-state oscillator strength $\omega$ with disappearing confinement, similar to the excitonic dependence in Fig. 4c. 


\section{Conclusion}

In a very simple model, we studied numerically the transition of one- and two-exciton energy and recombination spectra as a function of confinement volume (or area). The crossover is identified from the small-dot regime (with the excitons filling single-exciton states in accordance with effective Pauli exclusion principle inherited from constituent fermions) to the unconfined regime (with many excitons at falling into the same energy state).

\section{Acknowledgment}

The authors gratefully acknowledge discussions with J. Misiewicz and G. Sęk. Work supported by grant No. 2P03B08525 of the Ministry of Science and Higher Education (Poland).

\section{References}

[1] S. Okumura, T. Ogawa, Phys. Rev. B 65, 035105 (2002).

[2] M. Combescot, O. Betbeder-Matibet, Europhys. Lett. 59, 579 (2002).

[3] L.V. Keldysh, A.N. Kozlov, Zh. Eksp. Teor. Fiz. 54, 978 (1968) [Sov. Phys. JETP 27, 521 (1968)].

[4] E. Hanamura, H. Haug, Phys. Rep. 33, 209 (1977).

[5] L.V. Butov, A.C. Gossard, D.S. Chemla, Nature 418, 751 (2002).

[6] L. Jacak, P. Hawrylak, A. Wójs, Quantum Dots, Springer, Berlin 1998.

[7] A. Wójs, P. Hawrylak, Solid State Commun. 100, 487 (1996).

[8] S. Raymond, S. Fafard, P. Poole, A. Wójs, P. Hawrylak, S. Charbonneau, D. Leonard, R. Leon, P.M. Petroff, J.L. Merz, Phys. Rev. B 54, 11548 (1996).

[9] P. Podemski, G. Sek, R. Kudrawiec, A. Wójs, J. Misiewicz, B. Alloing, L.H. Li, A. Fiore, A. Somers, J.P. Reithmaier, A. Forchel, to be published. 